(c) 2010. The Korean Astronomical Society. All Rights Reserved.

\title{
SOME CURRENT ISSUES IN GALAXY FORMATION
}

\author{
Joseph SiLK \\ Beecroft Institute of Particle Astrophysics and Cosmology, Department of Physics, University of Oxford, \\ Denys Wilkinson Building 1 Keble Road Oxford, OX1 3RH \\ E-mail: silk@astro.ox.ac.uk \\ (Received June 17, 2010; Accepted August 11, 2010)
}

\begin{abstract}
The origin of the galaxies represents an important focus of current cosmological research, both observational and theoretical. Its resolution involves a comprehensive understanding of star formation and evolution, galaxy dynamics, supermassive black holes, and the cosmology of the very early universe. In this paper, I will review our current understanding of galaxy formation and review some of the challenges that lie ahead. Specific issues that I address include the galaxy luminosity function, feedback by supernovae and by AGN, and downsizing. I argue that current evidence favours two distinct modes of star formation in the early universe, in order to account for the origin of disk and massive spheroidal galaxies. However perhaps the most urgent need is for a robust theory of star formation.
\end{abstract}

key words: galaxies: formation; galaxies: evolution; cosmology: theory; stars: formation

\section{INTRODUCTION}

Here are some outstanding questions that pertain to galaxy formation. Can we account for the efficiency of star formation? Can we account for the star formation rate? Can we explain the galaxy luminosity function? Do we understand supermassive black hole feedback? The answer to these questions in all cases is no. But we should not despair. The missing link that yields a common thread to these questions is the need for a robust theory of the conversion of baryons into stars and into galaxies.

I review here some of the outstanding issues in our current understanding of galaxy formation. I address the efficiency of star formation in disk and in spheroidal galaxies, the star formation rate in galaxies both today and in the past, the luminosity function especially for faint and luminous galaxies (Figure 1), and the role of feedback by supernovae and supermassive black holes. I will argue that feedback in its diverse manifestations helps to partially resolve all of these issues. However much still remains to be done, both observationally and theoretically.

\section{IN THE BEGINNING...}

Star formation theory begins with the founder of the theory of gravitation. Isaac Newton realized in 1692 that fragmentation and subsequent star formation was inevitable in an infinite and initially homogeneous cloud. Gravity operated irreversibly and inevitably in accumulating matter around density fluctuations.

If the matter was evenly disposed throughout an infinite space, it could never convene into one mass; but some of it would convene into one mass and some into another, so as to make an infinite number of great masses, scattered at great distances from one to another throughout all that infinite space. And thus might the sun and fixed stars be formed, supposing the matter were of a lucid nature.

Newton's insight was remarkable. However he could not understand how gravity could differentiate between luminous bodies, or stars, and opaque bodies, or planets.

How the sun alone should be changed into a shin- 
ing body whilst all the planets continue opaque, or all they be changed into opaque ones whilst he remains unchanged, I do not think explicable by mere natural causes, but am forced to ascribe it to the counsel and contrivance of a voluntary Agent.

An intensely religious man, Newton gave up in despair at this point and appealed to a higher entity to come to the rescue.

James Jeans was not one to share this opinion, however. He developed fragmentation into quantitative physics. To him,

From the intrinsic evidence of his creation, the Great Architect of the Universe now begins to appear as a pure mathematician.

In 1902, he developed the theory of gravitational fragmentation which is now central to our understanding of star formation.

We have found that as Newton first conjectured, a chaotic mass of gas of approximately uniform density and of very great extent would be dynamically unstable: nuclei would tend to form in it, around which the whole matter would eventually condense. All celestial bodies originate by a process of fragmentation of nebulae out of chaos, of stars out of nebulae, of planets out of stars and satellites out of planets.

But Jeans did not solve the challenge posed by Newton of why stars as opposed to planets. The astronomer who faced this challenge was Arthur Eddington, who developed the theory of self-gravitating polytropic spheres in order to model stars. Simple stability considerations led him to realise that stars occupied a relatively narrow mass range. He showed in 1926 that star formation was inevitable.

Imagine a physicist calculating on a cloud-bound planet and ending with the dramatic conclusion, "What 'happens' is the stars."

\section{STAR FORMATION}

The Jeans mass sets the scale of fragmentation. It is defined to be the mass within a sphere of diameter the Jeans length, approximately the distance a sound wave crosses in a free fall time, and is proportional to $T^{3 / 2} \rho^{-1 / 2}$. At low densities, interstellar clouds radiate freely and are isothermal. During the isothermal

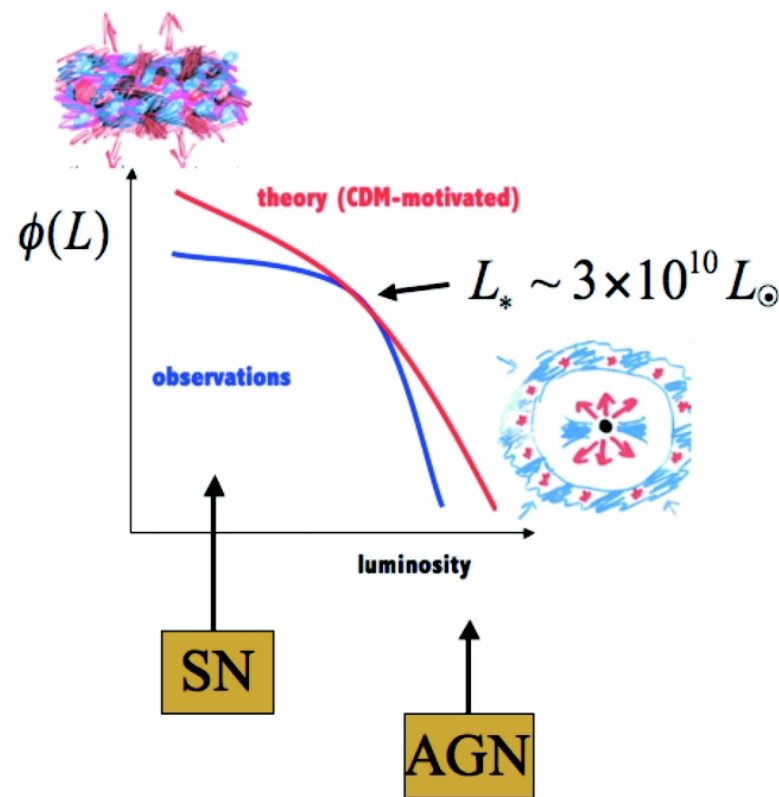

Fig 1. - The galaxy luminosity function: observation versus theory. The luminosity function is described by the Schechter function, an analytic fit to the data on nearby galaxies, whose shape is characterised by a typical luminosity $L_{*}$, above which the number density declines exponentially, and a powerlaw tail proportional to $L^{-\alpha}$, where $\alpha \approx 1$. The mass function is given by the Press-Schechter expression for the nonlinear evolution of self-gravitating spherical overdensities in an expanding universe, and is parametrized by a typical mass $M_{*}$ and low mass tail proportional to $M^{-2}$. One can identify a characteristic mass-to-luminosity at the one point where the two functions overlap, $M_{*} / L_{*}$.

phase of contraction, the Jeans mass decreases. Eventually the cloud becomes self-shielding, and the ensuing contraction is approximately adiabatic once the optical depth is large. The Jeans mass increases in this phase, which is the precursor to the phase of Kelvin-Helholtz contraction onto the stellar main sequence. The minimum opacity-limited Jeans mass is the fragmentation scale. It can be shown, quite insensitively to metallicity or dust content, to be $\alpha_{g}^{-3 / 2}$ solar masses, where $\alpha_{g}=G m_{p}^{2} / e^{2}$ is the so-called gravitational fine structure constant. This gives a minimum fragment mass of $\sim 0.003 \mathrm{M}_{\odot}$, a result that is found in essentially all numerical simulations of current epoch star formation. The dependence on temperature is approximately as $T^{1 / 4}$, and yields $\sim 0.01 \mathrm{M}_{\odot}$ for primordial abundances, 
appropriate to Population III. In general, fragmentation theory applied to a collapsing interstellar cloud implies that the minimum fragment mass is too small to be a star. Additional physics is needed.

A key addition is the accretion of cold gas. In the case of a singular isothermal sphere, accretion onto the core occurs at a rate $v_{s}^{3} / G$. In nearby cold molecular clouds, at $T \sim 10 \mathrm{~K}$, the inferred accretion rate is $\sim 10^{-6} \mathrm{M}_{\odot} / \mathrm{yr}$, and yields solar mass protostars on a time-scale of order the Kelvin-Helmholtz time. However in the case of the first stars, the presence of trace $H_{2}$ as a coolant means that the temperature $T \sim 10^{3} \mathrm{~K}$. Consequently, the accretion rate is $\sim 10^{-3} \mathrm{M}_{\odot} / \mathrm{yr}$, and one concludes that Population III stars, accreting over $10^{5}-10^{6} \mathrm{yr}$, had characteristic masses of $\sim 10^{2}-10^{3} \mathrm{M}_{\odot}$. Again, numerical simulations confirm this result.

However fragmentation and accretion do not suffice to reproduce the initial mass function of stars. A third process must be added, namely feedback, to halt the accretion, otherwise low mass stars would not form at present. In general, protostellar feedback halts collapse by tapping stellar gravitational energy via releasing magnetic energy. This simultaneously resolves the angular momentum problem, in that there is of the order of two orders of magnitude too much specific angular momentum in cloud cores to form stars directly.

One also requires magnetic feedback to account for the turbulence observed in cloud cores. It simultaneously results in inefficient star formation: were cores to collapse on a free fall time, one would have excessive star formation. Protostellar outflows are ubiquitous and provide momentum input by interactions of jets with the magnetized ISM. This suffices to prolong cloud longevity. In the case of massive clouds, OB stars provide feedback that ultimately disrupts the clouds. One observes over a wide range of molecular cloud masses that the star formation efficiency, defined to be star formation rate divided by gas mass and multiplied by cloud free fall time, is approximately 2 percent (Krumholz \& Tan, 2007). In this way one can arrive at a star formation rate for the Milky Way Galaxy (MWG) that is comparable to what is observed globally. In fact the Milky Way converts about $2 \%$ of its molecular gas content (approximately $3 \times 10^{9} \mathrm{M}_{\odot}$ ) into stars over cloud lifetimes of typically $\sim 10^{7} \mathrm{yr}$.

\section{FEEDBACK IN DISK GALAXIES}

It is at first sight rather remarkable that star formation in disk galaxies, both near and far, can be described by a simple law, with Star Formation Efficiency (SFE) being the controlling parameter:

$S F E=S F R \times R O T A T I O N T I M E / G A S M A S S=$ Constant.

The motivation comes from the gravitational instability of cold gas-rich disks, which provides the scaling, although the normalization depends on feedback physics. For the global law, in terms of star formation rate and gas mass per unit area, supernova regulation provides the observed efficiency of about $2 \%$ which fits essentially all local star-forming galaxies. One finds from simple momentum conservation that

$$
S F E=\frac{\sigma_{g a s} v_{\text {cool }} m * S N}{E_{S N}^{\text {initial }}} \approx 0.02 .
$$

This is a crude estimator of the efficiency of supernova momentum input into the interstellar medium but it reproduces the observed global normalization of the star formation law. The fit applies not only globally but to star formation complexes in individual galaxies such as M51 and also to starburst galaxies. This law is known as the Schmidt-Kennicutt law, and its application reveals that molecular gas is the controlling gas ingredient, and that in the outer parts of galaxies, where the molecular fraction is reduced due to the ambient UV radiation field and lower surface density, the star formation rate per unit gas mass also declines.

For disk instabilities to result in cloud formation, followed by cloud agglomeration and consequent star formation, one also needs to maintain a cold disk by accretion of cold gas. There is ample evidence of a supply of cold gas, for example in the M33 group. Other spiral galaxies show extensive reservoirs of $\mathrm{HI}$ in their outer regions. Recent data extend the SchmidtKennicutt law to $z \sim 2$. Remarkably an efficiency of $2.5 \%$ fits low and high redshift star-forming galaxies, with $S F E \propto S F R \times \Omega$, and starburst galaxies also lie on this relation (Genzel et al., 2010). There is a tendency for ultraluminous starbursts at $z \sim 2$ to have somewhat higher $S F E$.

\section{LUMINOSITY FUNCTION OF GALAXIES}

Theory provides the mass function of dark halos. Observation yields the luminosity function of galaxies, usually fit by a Schechter function. Comparison of 
the two is at first sight disconcerting. One can calculate the $M / L$ ratio for the two functions to overlap at one point, for a mass $M^{*}$ corresponding to $L_{*}$. Define $t_{c o o l}=\frac{\frac{3}{2} n k T}{\Lambda(T) n^{2}}$ and $t_{d y n}=\frac{3}{\sqrt{3} 2 \pi G \rho}$. For star formation to occur, cooling is essential, and the condition $t_{\text {cool }}<t_{d y n}$ guarantees cooling in an inhomogeneous galactic halo where gas clouds collide at the virial velocity. One finds that

$$
M_{\text {cool }}^{*}=\alpha^{3} \alpha_{g}^{-2} \frac{m_{p}}{m_{e}} \frac{t_{c o o l}}{t_{d y n}} T^{1+2 \beta} .
$$

For a cooling function $\Lambda(T) \propto T^{\beta}$, over the relevant temperature range $\left(10^{5}-10^{7} \mathrm{~K}\right)$, one can take $\beta \approx-1 / 2$ for a low metallicity plasma (Gnat \& Sternberg, 2007). The result is that one finds a characteristic galactic halo mass, in terms of fundamental constants, to be of order $10^{12} \mathrm{M}_{\odot}$. The inferred value of the mass-to-light ratio $M / L$ is similar to that observed for $L_{*}$ galaxies. This is a success for theory: dissipation provides a key ingredient in understanding the stellar masses of galaxies, at least for the "typical" galaxy. The characteristic galactic mass is understood by the requirement that cooling within a dynamical time is a necessary condition for efficient star formation. However the theory greatly overestimates galaxy numbers at low and high masses. Feedback is needed to address this problem.

\section{FEEDBACK IN LOW MASS GALAXIES}

Reionization gives an inevitable feedback for the lowest mass dwarfs. An abrupt increase of the sound speed to $\sim 10-20 \mathrm{~km} / \mathrm{s}$ at $\mathrm{z} \sim 10$ means that dwarfs of mass $10^{6}-10^{7} \mathrm{M}_{\odot}$ which have not yet collapsed and fragmented into stars will be disrupted. However more massive dwarfs are unaffected, as are the high $\sigma$ peaks that develop into early collapsing, but rare, low mass dwarfs. The accepted solution for gas disruption and dispersal in intermediate mass and massive dwarfs $\left(10^{8}-10^{10} \mathrm{M}_{\odot}\right)$ is by supernova feedback. Most gas is ejected by the first generations of supernovae for systems with escape velocity $\lesssim 50 \mathrm{~km} / \mathrm{s}$, leaving dim stellar remnants behind. This yields an acceptable fit to the low mass end of the galaxy luminosity function for the classical dwarfs.

One recent issue has emerged, however. The discovery of ultrafaint, low mass dwarfs in the MWG halo may be considered as an indication, and even verification, of a prediction of the supernova feedback hypoth- esis. The only worry is that a model tuned to fit more massive dwarfs such as the Magellanic Clouds is ineffective at dwarf disruption: the overabundance problem remains at the faint end of the luminosity function (Koposov et al., 2009). Semi-analytic galaxy formation models which fit the massive LMC-type dwarfs in the MWG and in M31 have inefficient feedback and overproduce the numbers of ultrafaint dwarfs. Conversely, models tuned to the ultrafaint dwarfs cannot account for the frequency of objects like the Magellanic Clouds. These are underproduced in the models as a consequence of excessive efficiency. This problem seems to be common to all semi-analytic galaxy formation models. Perhaps the loophole lies in small number statistics: with only two Magellanic Clouds one cannot draw overly far reaching conclusions. A similar comment applies to the M31 halo where there are also just two relatively massive dwarfs.

\section{FEEDBACK IN MASSIVE GALAXIES}

Supernovae cannot eject significant amounts of gas from massive galaxies. Baryons continue to be accreted over a Hubble time and the stellar mass grows. The consequences are that massive galaxies are overproduced in the models, and that the massive galaxies are too blue. Moreover the baryon fraction is typically only of order half of the primordial baryon fraction.

A clue towards a solution for these dilemmas comes from the accepted explanation of the Magorrian relation, which relates supermassive black hole mass to spheroid velocity dispersion. This requires collusion between black hole growth and the initial gas content of the galaxy when the old stellar spheroid formed. One conventionally appeals to outflows from the central black hole that deliver momentum to the protogalactic gas. When the black hole is sufficiently massive, the Eddington luminosity is high enough that residual gas is ejected. An estimate of the available momentum supply come from equating the Eddington momentum with self-gravity on circumgalactic gas shells,

$$
L_{E d d} / c=G M M_{g a s} / r^{2} .
$$

Blowout occurs and star formation terminates when the SMBH $-\sigma$ relation saturates. This occurs for $M_{B H} \propto$ $\sigma^{4}$, the observed slope, and gives, at least in order of magnitude, the correct normalisation of the relation.

There is also a role for AGN feedback at late epochs, when the so-called AGN radio mode heats halo gas, 


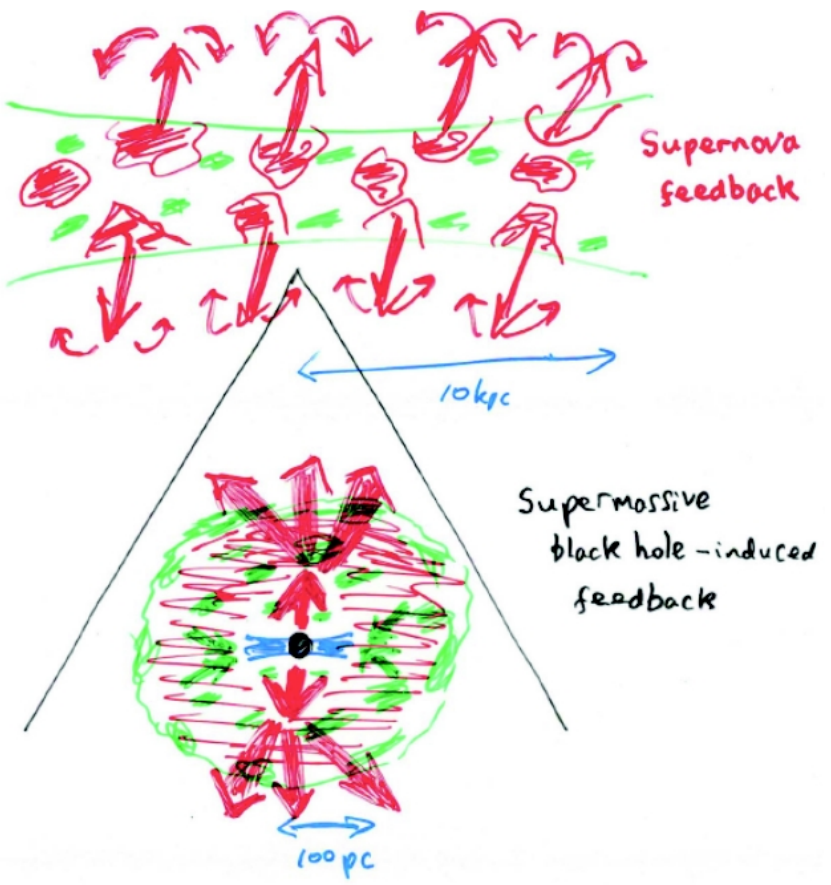

Fig 2. - The case for two modes of galaxy formation. Supernovae drive turbulence and fountains in star-forming disk galaxies, and are responsible for the low global star formation efficiency. Supermassive black holes quench star formation, accounting for the redness of elliptical galaxies, and may also play a role in triggering star formation in starbursts, thereby enhancing both the star formation efficiency and the specific star formation rate.

inhibits cooling, resolves the galaxy luminosity function bright end problem and accounts for the red colours of massive early-type galaxies. AGN feedback in the radio mode may also account for the suppression in numbers of intermediate mass and satellite galaxies. Feedback from AGN in the host galaxies preheats the halo gas that otherwise would be captured by satellites.

However reality may be not quite so simple. A more detailed examination suggests that negative feedback in momentum-driven winds by supermassive black holes falls short of explaining the observed $M_{B H}-\sigma$ correlation by a factor of a few (Silk \& Nusser, 2010). Moreover comparison of baryonic fractions with bulge-todisk ratios in nearby galaxies demonstrates that AGN alone do not eject significant amounts of baryons (Anderson \& Bregman, 2010). Something else seems to be needed.

\section{THE AGN-STAR FORMATION CONNECTION}

The most plausible addition to the physics is inclusion of star formation, induced and enhanced by the SMBH outflows. If AGN-driven outflows trigger star formation, the star formation rate is boosted by a factor $t_{d y n} / t_{j e t}$, and the outflow momentum is amplified by supernovae (Silk \& Norman, 2009). Consequently, the momentum supplied to the gas is boosted by the combination of AGN and star formation. There is extensive evidence, recently compiled by Netzer, that demonstrates the intimate connection of AGN luminosity and star formation rate over a wide dynamic range. Of course the causal direction is uncertain, and indeed the phenomena could be mutually self-regulating. To go beyond phenomenology, many details need to be refined, the most pressing perhaps being the nature of the black hole growth. However there are examples of jet-induced global star formation, as seen locally in Minkowski's object, and jet-induced $\mathrm{CO}$ formation (and excitation) at high redshift. CO is a prerequisite for star formation, and has been detected in large amounts in the host galaxies of high redshift quasars.

\section{MODES OF STAR FORMATION}

Incorporation of a positive role by AGN for star formation in extreme environments leads one to argue that a case can be made for two distinct feedback-regulated modes of star formation (Figure 2): at low redshift via supernovae and without $\mathrm{AGN}$, and at high redshift with triggering by AGN playing a central role. One would expect a transition between these two modes as the AGN duty cycle becomes shorter beyond $\mathrm{z} \sim 1$. Indeed a recent compilation (Gonzalez et al., 2010) of the specific star formation rate (SSR, or star formation rate per unit stellar mass) to $\mathrm{z} \sim 7$ in the GOODS field suggests that the star formation time-scale (or 1/SSR) goes from the MWG value of $\sim 3$ Gyr at low redshift to $\sim 0.5$ Gyr at $\mathrm{z} \gtrsim 2$.

There are two other transitions in this redshift range that may be relevant. At high redshift, major mergers between galaxies are common. Indeed the high redshift ULIRGs are invariably undergoing major gas-rich mergers. Theory suggests that at low redshift, gas accretion by cold streams is important, and that the cold streams are invariably clumpy and essentially indistinguishable from minor mergers of gas-rich dwarfs. In terms of the cosmic star formation history, normal star-forming galaxies dominate at low redshift $(\mathrm{z} \lesssim 2)$ 
whereas ULIRGs dominate at high redshift $(\mathrm{z} \gtrsim 2)$.

If the disk formation mode is distinct from the spheroid formation mode, then $\mathrm{SMBH}$ might be expected to show some reflection of alternative growth histories. So-called pseudobulges form from secular instability of disks and contain smaller $\mathrm{SMBH}$ than do the more massive bulges that may have formed via major gas-rich mergers. It is interesting that $\mathrm{SMBH}$ in pseudobulges lie low on the Magorrian relation (Kormendy \& Tremaine, private communication), possibly reflecting the different black hole formation histories and the associated distinct star formation modes. Recent data on $z \sim 6$ quasars suggest that the most massive black holes indeed lie high on the black hole/dynamical mass relation.

Much work needs to be done to see whether allowance for two modes of star formation can help resolve some of the outstanding problems in galaxy formation. Perhaps the greatest challenge in any combination of cold stream/minor merger/major merger scenario for gas delivery to drive both star formation and SMBH feeding is that $\sim 16 \%$ of nearby galactic disks are bulgeless. In addition to the many uncertainties in star formation theory (and I have not addressed one of the key issues, that of the IMF), there remains the nature of black hole growth. Whether the black holes grow by gas accretion, in which case feedback may play a role in angular momentum transfer (Antonuccio-Deloglu \& Silk, 2010), or by mergers, or by an appropriate combination, remains unresolved.

\section{CONCLUSIONS}

Cold gas flows via filaments/minor mergers lead to disk and bulge formation. Supernovae drive turbulence and fountains in star-forming disk galaxies, and are responsible for the low global star formation efficiency. Major mergers along with hot gas infall followed by cooling forms massive spheroids at high efficiency. The role of SMBH is to quench star formation at early epochs, thereby accounting for the redness of elliptical galaxies, and to heat intracluster gas at late epochs, thereby preventing gas cooling and late star formation. Of course this process cannot be completely efficient, and indeed $30 \%$ of nearby ellipticals have modest amounts of ongoing star formation. More speculatively, SMBH may also play a role in triggering star formation in starbursts, especially in ULIRGs. Positive SMBH feedback can enhance both the star formation efficiency and the specific star formation rate.

The origin of SMBH remains a mystery and must certainly play a key role in ascertaining the detailed nature of SMBH feedback. Improved resolution in theory and observation is needed. The great projects of the future, including the ELTs, JWST and LSST, will surely play key roles in this endeavour.

\section{REFERENCES}

Anderson, M. \& Bregman, J., 2010, Do Hot Halos Around Galaxies Contain the Missing Baryons?, ApJ, 714, 320

Antonuccio-Deloglu, V. \& Silk, J., 2010, Active Galactic Nuclei Activity: Self-Regulation from Backflow, MNRAS, 405, 1303

Genzel, R., et al., 2010, A Study of the Gas-Star Formation Relation over Cosmic Time, MNRAS, submitted, arXiv:1003.5180

Gnat, O. \& Sternberg, A., 2007, Time-Dependent Ionization in Radiatively Cooling Gas, ApJS, 168, 213

Gonzalez, V., et al., 2010, The Stellar Mass Density and Specific Star Formation Rate of the Universe at $\mathrm{z} \sim 7$, ApJ, 713, 115

Koposov, S., et al., 2009, A Quantitative Explanation of the Observed Population of Milky Way Satellite Galaxies, ApJ, 696, 2179

Kormendy, J. \& Tremaine, S., private communication Krumholz, M. \& Tan, J., 2007, Slow Star Formation in Dense Gas: Evidence and Implications, ApJ, 654,304

Silk, J. \& Norman, C., 2009, Global Star Formation Revisited, ApJ, 700, 262

Silk, J. \& Nusser, A., 2010, Momentum-Driven Winds and Positive AGN Feedback, MNRAS, submitted, arXiv:1004.0875 